\title{
Characteristics, Prognostic Factors, and Survival of Patients with NK/T-Cell Lymphoma of Non-upper Aerodigestive Tract: A 17-Year Single-Center Experience
}

\section{Ze-Long Liu, MD ${ }^{1}$ \\ $\mathrm{Xi}-\mathrm{Wen} \mathrm{Bi}, \mathrm{MD}^{1}$ \\ Xue-Wen Zhang, MD ${ }^{1}$ \\ De-Xin Lei, MD' \\ Pan-Pan Liu, MD ${ }^{1}$ \\ Hang Yang, MD' \\ Yan Gao, MD' \\ Yuan-Xue Jiang, MD2 \\ Wen-Oi Jiang, $M D^{1}$ \\ Yi Xia, MD 1}

${ }^{1}$ Department of Medical Oncology, Sun Yat-sen University Cancer Center, State Key Laboratory of Oncology in South China, Collaborative Innovation Centre of Cancer Medicine, Guangzhou, ${ }^{2}$ Department of Oncology, Pan Yu Central Hospital, Guangzhou, China

Correspondence: Wen-Qi Jiang, MD

Department of Medical Oncology,

Sun Yat-sen University Cancer Center,

State Key Laboratory of Oncology in

South China, Collaborative Innovation Centre of Cancer Medicine, 651 Dongfeng East Road, Guangzhou 510060, China

Tel: 86-20-87343352

E-mail: jiangwenqi111@163.com

Co-correspondence: Yi Xia, MD

Department of Medical Oncology,

Sun Yat-sen University Cancer Center,

State Key Laboratory of Oncology in

South China, Collaborative Innovation Centre of Cancer Medicine, 651 Dongfeng East Road, Guangzhou 510060, China

Tel: 86-20-87333359

E-mail: xiayi@sysucc.org.cn

Received December 8, 2018

Accepted March 28, 2019

Published Online April 1, 2019

*Ze-Long Liu, Xi-Wen Bi, and Xue-Wen Zhang contributed equally to this work.

\section{Purpose}

The extranodal natural killer (NK)/T-cell lymphoma (NKTCL) of non-upper aerodigestive tract (NUAT) was found to have clinical heterogeneity compared with NKTCL of the upper aerodigestive tract (UAT) in small scale studies. We conducted this study in a much larger cohort to analyze the clinical characteristics, prognostic factors, treatment modality, and clinical outcomes of patients with NUAT-NKTCL.

\section{Materials and Methods}

From January 2001 to December 2017, a total of 757 NKTCL patients were identified and included in this study, including 92 NUAT-NKTCL patients $(12.2 \%)$ and 665 UAT-NKTCL patients (87.8\%).

\section{Results}

NUAT-NKTCL patients had relatively poorer performance status, more unfavorable prognostic factors, and more advanced stage, compared with UAT-NKTCL patients. The 5-year overall survival (OS) was $34.7 \%$ for NUAT-NKTCL, which was significantly worse than UAT-NKTCL $(64.2 \%, p<0.001)$. The median OS duration was 30.9 months for NUAT-NKTCL. Multivariate analysis showed that presence with B symptoms and elevated serum lactate dehydrogenase independently predicted worse OS. International prognostic index score and prognostic index of NK lymphoma score still had prognostic values in NUAT-NKTCL, while the Ann Arbor system could not accurately predict the OS.

\section{Conclusion}

NUAT-NKTCL is a distinctive subtype of NKTCL in many aspects. Patients with NUAT-NKTCL have relatively poorer performance status, more unfavorable prognostic factors, more advanced stage, and poorer prognosis.

\section{Key words}

Extranodal NK-T-cell lymphoma, Non-upper aerodigestive tract, Prognostic factors, Survival 


\section{Introduction}

Extranodal natural killer (NK) / T-cell lymphoma (NKTCL) is a distinctive clinicopathological entity of non-Hodgkin's lymphoma according to the World Health Organization (WHO) classification of lymphoid neoplasms [1]. The disease is much more common in Asian and Latin American populations, and mostly happens in middle-aged male, with clinical characteristics of necrosis, ulceration, and destructive lesion [1]. NKTCL shows typical pathological features of angiocentric lesion, vessel damage, and coagulative necrosis [1].

NKTCL mostly originate in the nasal cavity or other sites of the upper aerodigestive tract (NK/T-cell lymphoma of upper aerodigestive tract [UAT-NKTCL]), while it could also happen in diverse sites outside the UAT (NK/T-cell lymphoma of non-upper aerodigestive tract [NUAT-NKTCL]), such as the skin and soft tissue, gastrointestinal tract (GI tract), liver, spleen, testicle, lung, eye, adrenal gland, and so forth [2,3]. Previous retrospective studies found obvious heterogeneity in aspects of clinical features, risk factors, treatment response and prognosis between UAT-NKTCL and NUAT-NKTCL [2-10]. According to the research, NUATNKTCL patients had more advanced clinical stage, more unfavorable prognostic factors, worse treatment response, and clinical outcomes compared with UAT-NKTCL patients. Thus, NKTCL was divided into two subtypes in the WHO classification of tumors of lymphoid tissues (2008): nasal NKTCL (including UAT-NKTCL beyond the nasal cavity) and extranasal NKTCL (excluding UAT-NKTCL).

Currently, treatment experience of NKTCL is based on previous researches of UAT-NKTCL. UAT-NKTCL is highly sensitive to radiation therapy, which is now the main treatment for patients with limited stage disease. Published literature found unsatisfactory results of anthracyclines-based chemotherapy regimens in UAT-NKTCL, but conversely, promising results of asparaginase (ASP)-containing chemotherapy regimens [11-13]. Due to the much lower incidence and limited clinical evidence, treatment modality for NUATNKTCL remains to be elucidated so far. A retrospective study containing 59 patients with NUAT-NKTCL in 2008 analyzed clinical data and emphasized the clinical heterogeneity in aspects of clinical features, prognostic factors, and survival in the anthracycline era [3]. Thus, we conducted this retrospective study in a cohort of 92 NUAT-NKTCL patients, aiming to analyze the clinical characteristics, prognostic factors, treatment, and survival of these patients in the nonanthracycline era.

\section{Materials and Methods}

\section{Patient selection and staging evaluation}

We retrospectively reviewed the medical records patients who were diagnosed with NKTCL at Sun Yat-sen University Cancer Center between 2001 and 2017. The inclusion criteria for our study were as follows: (1) patients confirmedly diagnosed with NKTCL based on the WHO classification of lymphomas [1]; (2) complete follow-up data. The term UAT used in this study included the nasal cavity, Waldeyer's ring, hypopharynx, larynx, and oral cavity. And NUAT-NKTCL represents NKTCL that originates beyond these anatomical sites. In total of 757 NKTCL patients were identified, including 92 NUAT-NKTCL patients (12.2\%) and 665 UAT-NKTCL patients $(87.8 \%)$.

The initial clinical evaluation included the following: a complete history and physical examination; serum biochemistry, including serum lactate dehydrogenase (LDH) levels; a bone marrow examination; and magnetic resonance imaging and/or computed tomography of the head, neck, chest, abdomen, and pelvis. Additional baseline evaluations, including positron emission tomography-computed tomography and plasma Epstein-Barr virus DNA (EBV-DNA) copy number, were performed in selected patients if necessary. Clinical staging was performed based on the Ann Arbor staging system. The international prognostic index (IPI) and the prognostic index of natural killer lymphoma (PINK) were calculated for all patients at initial presentation.

\section{Response criteria and statistical analysis}

Treatment response was assessed according to the response criteria for lymphomas from the International Harmonization Project on Lymphoma [14]. The overall survival (OS) was calculated from the date of diagnosis to the date of death from any cause or the date of the last follow-up visit. The progression-free survival (PFS) was measured from the date of diagnosis to the date of disease progression, relapse, death from any cause, or the last follow-up visit. Categorical variables were compared using the chi-square tests or Fisher exact tests, while continuous variables were compared using the Mann-Whitney U tests. Survival analysis was performed by plotting the Kaplan-Meier survival curve, in which a logrank test was used to detect a statistically significant difference. The Cox proportional hazard model was used for a univariate screen of all potential predictors of survival. Variables with statistical and clinical significance were included in the multivariate analysis using the stepwise forward Cox regression model. A two-sided p-value $<0.05$ was considered significant. The statistical analysis was performed using 
Table 1. The clinical characteristics of patients with NUAT-NKTCL and UAT-NKTCL

\begin{tabular}{|c|c|c|c|}
\hline Parameter & NUAT & UAT & p-value \\
\hline Overall & $92(100)$ & $665(100)$ & \\
\hline \multicolumn{4}{|l|}{ Sex } \\
\hline Male & $58(63.0)$ & $463(69.6)$ & 0.202 \\
\hline Female & $34(37.0)$ & $202(30.4)$ & \\
\hline \multicolumn{4}{|l|}{ Age (yr) } \\
\hline Median (range) & $35(1-79)$ & $43(3-80)$ & 0.004 \\
\hline$\leq 60$ & $80(87.0)$ & $583(87.7)$ & 0.846 \\
\hline$>60$ & $12(13.0)$ & $82(12.3)$ & \\
\hline \multicolumn{4}{|l|}{ ECOG score } \\
\hline $0-1$ & $53(57.6)$ & $577(86.8)$ & $<0.001$ \\
\hline$\geq 2$ & $39(42.4)$ & $69(10.4)$ & \\
\hline Unknown & 0 & $19(2.9)$ & \\
\hline B symptoms ${ }^{\text {a) }}$ & $48(52.7)$ & $344(52.4)$ & 0.956 \\
\hline Involvement of regional lymph nodes ${ }^{\text {b) }}$ & $45(51.7)$ & $242(36.9)$ & 0.008 \\
\hline Involvement of distant lymph nodes ${ }^{c)}$ & $25(28.1)$ & $64(9.8)$ & $<0.001$ \\
\hline Extranodal sites involvement $\geq 2^{\mathrm{d})}$ & $40(44.0)$ & $81(12.4)$ & $<0.001$ \\
\hline Involvement of bone marrow ${ }^{\mathrm{d})}$ & $10(11.0)$ & $13(2.0)$ & $<0.001$ \\
\hline Presence of hemophagocytosis & $7(7.6)$ & $38(5.7)$ & 0.471 \\
\hline \multicolumn{4}{|l|}{ Ann Arbor stage } \\
\hline I-II & $32(34.8)$ & $557(83.8)$ & $<0.001$ \\
\hline III-IV & $59(64.1)$ & $99(14.9)$ & \\
\hline Unknown & $1(1.1)$ & $9(1.4)$ & \\
\hline Elevated $\mathrm{LDH}^{\mathrm{e})}$ & $41(55.4)$ & $169(28.5)$ & $<0.001$ \\
\hline \multicolumn{4}{|l|}{ EBV DNA in blood } \\
\hline Detectable & $28(30.4)$ & $216(32.5)$ & 0.093 \\
\hline Undetectable & $15(16.3)$ & $166(25.0)$ & \\
\hline Unknown & $49(53.3)$ & $283(42.6)$ & \\
\hline Median Ki-67 index (range, $\%)^{\mathrm{f}}$ & $70(15-100)$ & $60(5-100)$ & $<0.001$ \\
\hline Median SUVmax (range)g) & $14.6(2.7-29.3)$ & $13.9(2.0-79.4)$ & 0.924 \\
\hline \multicolumn{4}{|l|}{ IPI score } \\
\hline $0-1$ & $21(22.8)$ & $476(71.6)$ & $<0.001$ \\
\hline 2 & $19(20.7)$ & $36(5.4)$ & \\
\hline 3 & $28(30.4)$ & $46(6.9)$ & \\
\hline $4-5$ & $12(13.0)$ & $27(4.1)$ & \\
\hline Unknown & $12(13.0)$ & $80(12.0)$ & \\
\hline \multicolumn{4}{|l|}{ PINK score } \\
\hline 0 & 0 & $475(71.4)$ & $<0.001$ \\
\hline 1 & $24(26.1)$ & $104(15.6)$ & \\
\hline 2 & 33 (35.9) & $62(9.3)$ & \\
\hline $3-4$ & $33(35.9)$ & $11(1.7)$ & \\
\hline Unknown & $1(1.1)$ & $13(2.0)$ & \\
\hline
\end{tabular}

(Continued to the next page)

the Statistical Package for the Social Sciences (SPSS) ver. 24.0 software (IBM Corp., Armonk, NY).

\section{Ethical statement}

The study was approved by the ethics committee of Sun Yat-sen University Cancer Center (No.YB-201812) and the informed consent for collection and publication of medical 
Table 1. Continued

\begin{tabular}{lcc} 
Parameter & NUAT & UAT \\
Treatment modalities & & $193(29.0)$ \\
CT alone & $64(69.6)$ & $19(2.9)$ \\
RT alone & 0 & $309(46.5)$ \\
CT + RT & $19(20.7)$ & $17(2.6)$ \\
RT + CT & $3(3.3)$ & $114(17.1)$ \\
Sandwich chemoradiation & $4(4.3)$ & $13(2.0)$ \\
\hline Supportive care & $2(2.2)$ & 0.001 \\
\hline
\end{tabular}

Values are presented as number $(\%)$. NUAT, non-upper aerodigestive tract; NKTCL, natural killer/T-cell lymphoma; UAT, upper aerodigestive tract; ECOG, Eastern Cooperative Oncology Group; LDH, lactate dehydrogenase; EBV, Epstein-Barr virus; SUVmax, the maximal standardized uptake value; IPI, International Prognostic Index; PINK, Prognostic Index of Nat-

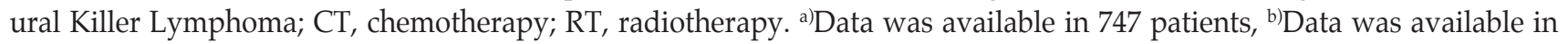
742 patients, ') Data was available in 743 patients, ${ }^{\text {d) }}$ Data was available in 745 patients, e)Data was available in 667 patients, ${ }^{\mathrm{f})}$ Data was available in 519 patients, ${ }^{\mathrm{g}}$ )Data was available in 318 patients.

information was obtained from all study patients at their first visit to our hospital. The authenticity of this article has been validated by uploading the key raw data onto the Research Data Deposit public platform (www.researchdata.org.cn), with the approval RDD number as RDDA2019001016.

\section{Results}

\section{Clinical characteristics}

The clinical characteristics of the NUAT-NKTCL patients and UAT-NKTCL patients are presented in Table 1 . Both NUAT-NKTCL and UAT-NKTCL exhibited a male predominance, with similar male to female ratios of 1.7:1 and 2.3:1, respectively. The median age of NUAT-NKTCL and UATNKTCL was 35 years (range, 1 to 79 years) and 43 years (range, 3 to 80 years), which was significantly different ( $\mathrm{p}=$ 0.004). However, there were $12(13.0 \%)$ and $82(12.3 \%)$ patients older than 60 years in two groups, respectively, which was not significantly different $(\mathrm{p}=0.846)$.

Compared with UAT-NKTCL, more NUAT-NKTCL patients had a poor performance status (Eastern Cooperative Oncology Group [ECOG] score $\geq 2,42.4 \%$ vs. 10.7\%, p < 0.001). More NUAT-NKTCL patients presented with involvement of regional lymph nodes ( $51.7 \%$ vs. $36.9 \%, \mathrm{p}=0.008)$, involvement of distant lymph nodes $(28.1 \%$ vs. $9.8 \%, \mathrm{p}<0.001)$, two or more extranodal lesions ( $44.0 \%$ vs. $12.4 \%$, p $<0.001)$, involvement of bone marrow (11.0\% vs. $2.0 \%, \mathrm{p}<0.001)$, and elevated LDH levels (55.4\% vs. $28.5 \%$, p < 0.001). Conversely, there were not significant differences between two groups in terms of presence of B symptoms, presence of hemophagocytosis, and detectable EBV DNA in blood. The median of Ki-67 index in NUAT-NKTCL patients was slightly higher than that in UAT-NKTCL patients $(70 \%$ vs. $60 \%, \mathrm{p}<0.001)$. The median value of baseline maximal standardized uptake value in positron emission tomography for primary lesion was similar in NUAT-NKT-CL and UAT-NKTCL patients (2.7 vs. 2.0, $\mathrm{p}=0.924)$. NUAT-NKTCL patients had more advanced stage (stage III/IV, $64.8 \%$ vs. $15.1 \%$, p < 0.001), higher IPI scores (50.0\% vs. $12.5 \%$ with IPI $\geq 3, p<0.001)$, and higher PINK scores (36.3\% vs. $1.7 \%$ with PINK $\geq 3, \mathrm{p}<0.001$ ), compared with UAT-NKTCL patients.

The most common site of primary lesions in NUAT-NKTCL was the skin and soft tissue $(n=42,45.7 \%)$, followed by the GI tract $(n=18,19.6 \%)$, and the eye and adnexa $(n=14$, $15.2 \%$ ) (Table 2). Other sites of primary disease included the testicle and ovary $(n=5,5.4 \%)$, the spleen $(n=4,4.3 \%)$, the lung and trachea $(n=2,2.2 \%)$, the central nervous system $(n=2,2.2 \%)$, and the adrenal gland $(n=2,2.2 \%)$. The primary lesions were unable to be classified in three patients $(3.3 \%)$ due to the extensive involvement of multiple systems at the initial presentation.

\section{Treatment and response}

The treatment modalities for NUAT-NKTCL patients depended on their physicians and considered, e.g., the patient's physical status, clinical presentation, and comorbidities. Initial treatment modalities for this cohort are summarized in Table 2. The primary treatment included chemotherapy (CT) alone (64 cases, 69.6\%), CT followed by radiotherapy (RT; 19 cases, 20.7\%), RT followed by CT (3 cases, 3.3\%), sandwich chemoradiation (4 cases, $4.3 \%$ ), and supportive 
Table 2. Treatment modalities of patients with NK/T-cell lymphoma of non-upper aerodigestive tract

\begin{tabular}{|c|c|c|c|c|c|c|}
\hline Treatment & Total & CT alone & $\mathrm{CT}+\mathrm{RT}$ & $\mathrm{RT}+\mathrm{CT}$ & $\begin{array}{c}\text { Sandwich } \\
\text { chemoradiation }\end{array}$ & $\begin{array}{l}\text { Supportive } \\
\text { care }\end{array}$ \\
\hline All patients & $92(100)$ & $64(69.6)$ & $19(20.7)$ & $3(3.3)$ & $4(4.3)$ & $2(2.2)$ \\
\hline Stage I-II & $32(100)$ & $16(50.0)$ & $13(40.6)$ & $1(3.1)$ & $1(3.1)$ & $1(3.1)$ \\
\hline Stage III-IV & $59(100)$ & $47(79.7)$ & $6(10.2)$ & $2(3.4)$ & $3(5.1)$ & $1(1.7)$ \\
\hline Unknown & $1(100)$ & $1(100)$ & 0 & 0 & 0 & 0 \\
\hline \multicolumn{7}{|l|}{ Primary sites } \\
\hline Skin and soft tissue & $42(100)$ & $26(61.9)$ & $11(26.2)$ & $2(4.8)$ & $3(7.1)$ & 0 \\
\hline Gastrointestinal tract & $18(100)$ & $16(88.9)$ & $1(5.6)$ & 0 & 0 & $1(5.6)$ \\
\hline Eye and adnexa & $14(100)$ & $7(50.0)$ & $6(42.9)$ & 0 & 0 & $1(7.1)$ \\
\hline Spleen & $4(100)$ & $4(100)$ & 0 & 0 & 0 & 0 \\
\hline Testicle and ovary & $5(100)$ & $4(80.0)$ & 0 & 0 & $1(20.0)$ & 0 \\
\hline Adrenal gland & $2(100)$ & $1(50.0)$ & $1(50.0)$ & 0 & 0 & 0 \\
\hline Central nervous system & $2(100)$ & $1(50.0)$ & 0 & $1(50.0)$ & 0 & 0 \\
\hline Lung and trachea & $2(100)$ & $2(100)$ & 0 & 0 & 0 & 0 \\
\hline Unclassifiable & $3(100)$ & $3(100)$ & 0 & 0 & 0 & 0 \\
\hline
\end{tabular}

Values are presented as number $(\%)$. NK, natural killer; CT, chemotherapy; RT, radiotherapy.

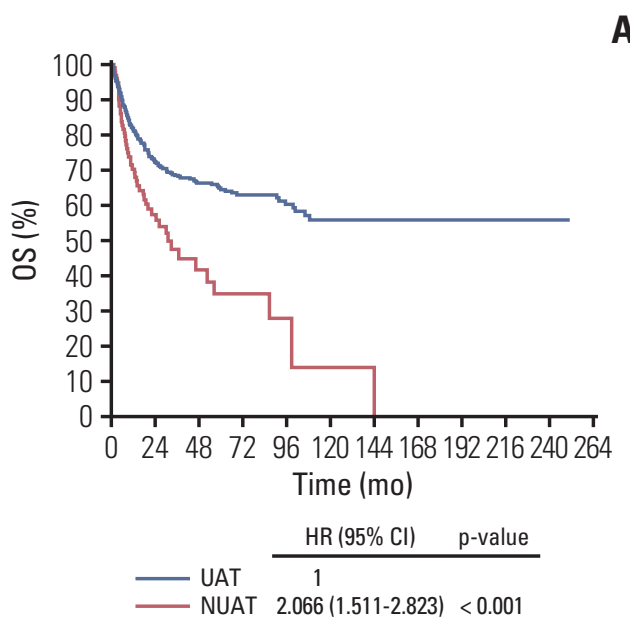

A

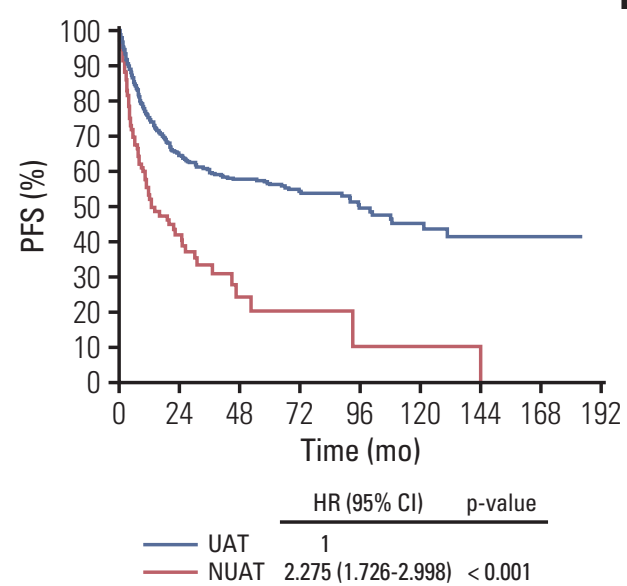

Fig. 1. Kaplan-Meier plots of overall survival (OS) (A) and progression-free survival (PFS) (B) for non-upper aerodigestive tract (NUAT) - natural killer/ T-cell lymphoma (NKTCL) and upper aerodigestive tract (UAT)-NKTCL. HR, hazard ratio; CI, confidence interval.

care alone (2 cases, 2.2\%). RT was applied to $46.9 \%(15 / 32)$ of limited-stage (stage I/II) patients but only $18.6 \%(11 / 59)$ of advanced-stage patients, which was significantly different $(\mathrm{p}<0.001)$. Eighty-four point six percent of patients $(n=22)$ receiving RT had their primary tumor located in skin and soft tissue, and eye and its adnexa.

Chemotherapy regimens varied during the study period depending upon the treating physicians (S1 Table). The most frequently used regimens included L-asparaginase (L-ASP) or pegaspargase/gemcitabine/ oxaliplatin (GELOX/PGEMOX) in 29 patients (32.2\%), cyclophosphamide/doxorubicin/vincristine/ prednisone (CHOP) in 10 patients $(11.1 \%)$, etoposide/doxorubicin/vincristine/cyclophosphamide/pre dnisone (EPOCH) in 15 patients $(16.7 \%)$, CHOP plus L-ASP (CHOP-L) in seven patients $(7.8 \%)$, and miscellaneous regimens in 29 patients $(32.2 \%)$. In summary, ASP was administered as part of the chemotherapy in 62 of 90 patients $(68.9 \%)$. Details in dosage of chemotherapeutic regimens were for- 
Table 3. Univariate analysis of prognostic factors in patients with NK/T-cell lymphoma of non-upper aerodigestive tract

\begin{tabular}{|c|c|c|c|c|}
\hline \multirow{2}{*}{ Variable } & \multicolumn{2}{|l|}{ OS } & \multicolumn{2}{|l|}{ PFS } \\
\hline & HR $(95 \%$ CI $)$ & p-value & HR $(95 \%$ CI) & p-value \\
\hline Sex (female vs. male) & $0.730(0.394-1.351)$ & 0.316 & $0.735(0.430-1.258)$ & 0.261 \\
\hline Age ( $>60$ yr vs. $\leq 60 \mathrm{yr})$ & $1.482(0.657-3.341)$ & 0.343 & $1.024(0.483-2.168)$ & 0.951 \\
\hline ECOG score ( $\geq 2$ vs. $0-1$ ) & $1.219(0.690-2.154)$ & 0.495 & $1.432(0.870-2.359)$ & 0.158 \\
\hline Stage (III-IV vs. I-II) & $1.457(0.876-2.423)$ & 0.147 & $1.012(0.639-1.604)$ & 0.958 \\
\hline B symptoms (yes vs. no) & $2.019(1.100-3.703)$ & 0.023 & $1.902(1.128-3.207)$ & 0.016 \\
\hline LDH (elevated vs. normal) & $2.396(1.198-4.789)$ & 0.013 & $1.458(0.822-2.587)$ & 0.197 \\
\hline Involvement of regional lymph nodes & $1.344(0.736-2.454)$ & 0.336 & $0.998(0.591-1.687)$ & 0.995 \\
\hline Involvement of distant lymph nodes & $1.521(0.790-2.928)$ & 0.209 & $1.127(0.634-2.006)$ & 0.683 \\
\hline Extranodal sites involvement ( $\geq 2$ vs. $0-1$ ) & $1.974(1.094-3.564)$ & 0.024 & $1.263(0.758-2.105)$ & 0.371 \\
\hline Involvement of bone marrow & $2.014(0.842-4.817)$ & 0.116 & $1.497(0.677-3.312)$ & 0.319 \\
\hline Presence of hemophagocytosis & $4.363(1.776-10.717)$ & 0.001 & $2.766(1.156-6.616)$ & 0.022 \\
\hline IPI score (3-5 vs. $0-2)$ & $2.850(1.490-5.451)$ & 0.002 & $1.498(0.870-2.580)$ & 0.145 \\
\hline PINK score (3-4 vs. 1-2) & $2.065(1.133-3.764)$ & 0.018 & $1.153(0.681-1.953)$ & 0.595 \\
\hline Treatment (RT vs. no RT) & $0.634(0.328-1.224)$ & 0.174 & $0.478(0.265-0.861)$ & 0.014 \\
\hline $\begin{array}{l}\text { Chemotherapeutic regimens } \\
\text { (ASP-containing vs. ASP-absent) }\end{array}$ & $0.601(0.332-1.089)$ & 0.093 & $0.673(0.397-1.142)$ & 0.142 \\
\hline Treatment response (CR vs. no CR) & $0.289(0.151-0.553)$ & $<0.001$ & $0.200(0.111-0.358)$ & $<0.001$ \\
\hline
\end{tabular}

NK, natural killer; OS, overall survival; PFS, progression-free survival; HR, hazard ratio; CI, confidence interval; ECOG, Eastern Cooperative Oncology Group; LDH, lactate dehydrogenase; IPI, International Prognostic Index; PINK, Prognostic Index of Natural Killer Lymphoma; RT, radiotherapy; ASP, asparaginase; CR, complete remission.
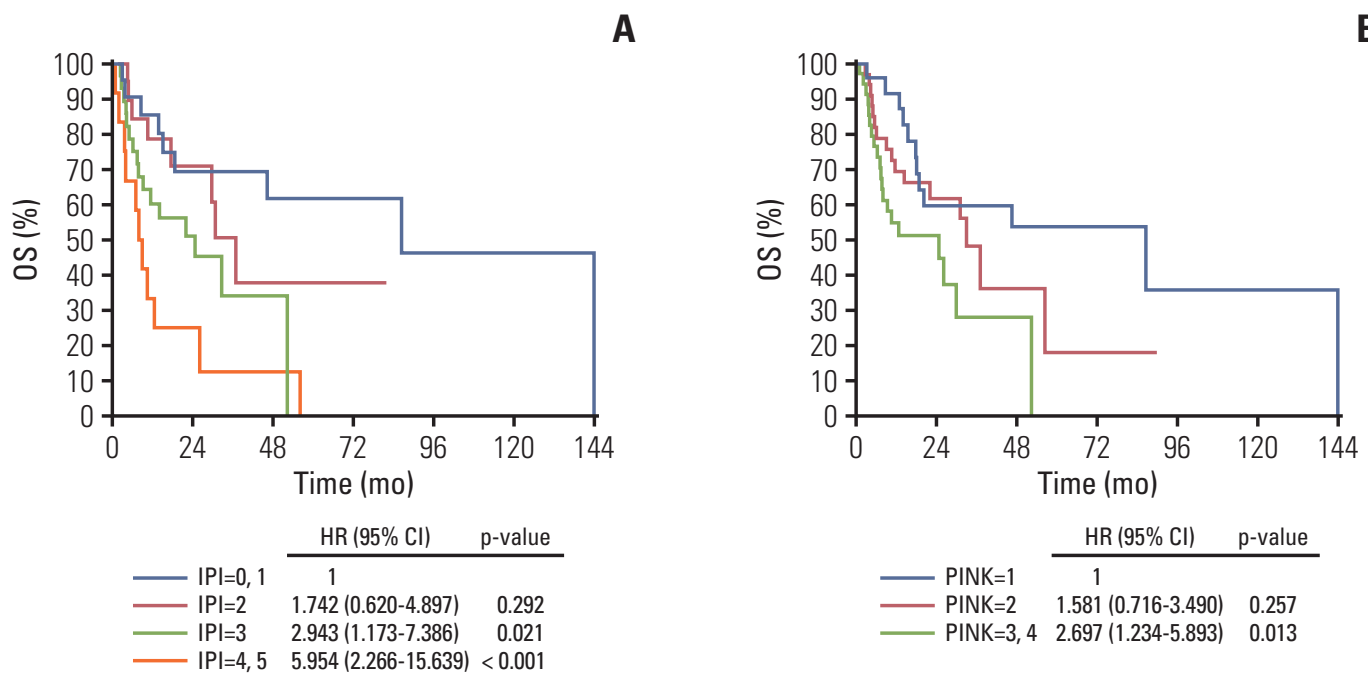

Fig. 2. Kaplan-Meier plots of overall survivals (OS) for non-upper aerodigestive tract-natural killer/T-cell lymphoma by the International Prognostic Index (IPI) score (A) and the Prognostic Index of Natural Killer Lymphoma (PINK) score (B). $\mathrm{HR}$, hazard ratio; $\mathrm{CI}$, confidence interval. 


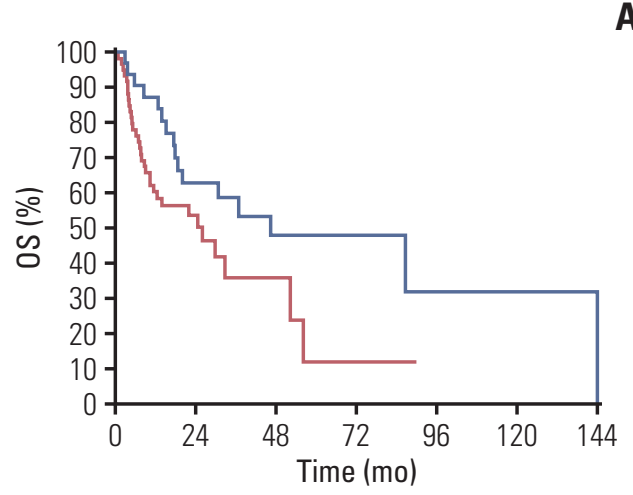

A
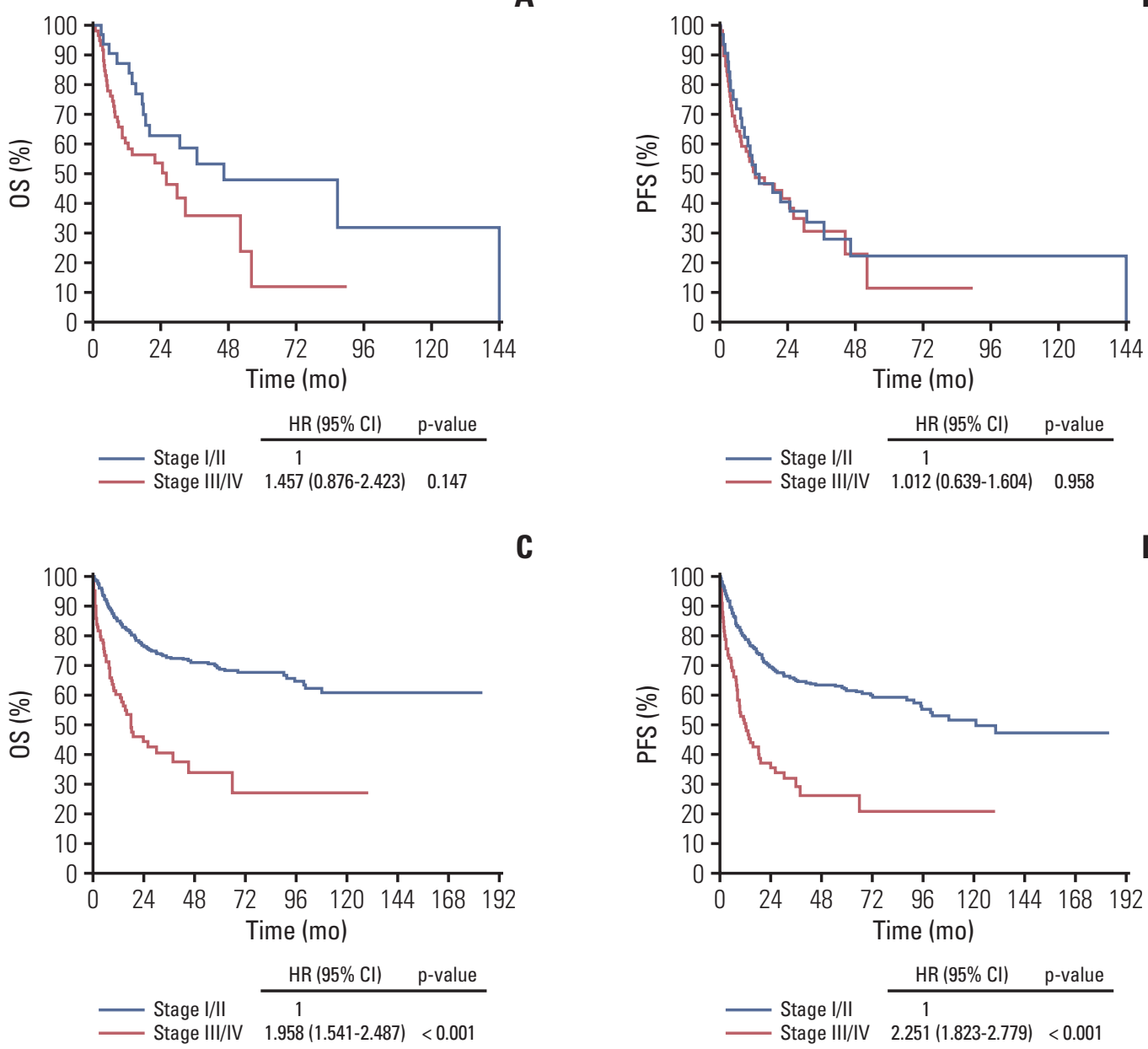

Fig. 3. Kaplan-Meier plots of overall survival (OS) (A) and progression-free survival (PFS) (B) for non-upper aerodigestive tract-natural killer/T-cell lymphoma (NKTCL) by Ann Arbor stage; and Kaplan-Meier plots of OS (C) and PFS (D) for upper aerodigestive tract-NKTCL by Ann Arbor stage. HR, hazard ratio; CI, confidence interval.

merly reported [15]. The median number of chemotherapy cycles was 4 (range, 0 to 12). The clinical characteristics of NUAT-NKTCL patients treated with ASP-containing and ASP-absent chemotherapy regimens were compared in S2 Table.

A total of 26 NUAT-NKTCL patients (28.3\%) received RT for primary treatment, which was significantly less than UAT-NKTCL patients $(69.0 \%, \mathrm{p}<0.001)$. RT was administered as a consolidative therapy following complete remission (CR) to $\mathrm{CT}$ in nine patients with radiation fields covering the primary area of disease, 12 patients received RT to the residual lesions after partial remission (PR) to $\mathrm{CT}$, and 2 patients received RT after stable disease (SD) to CT. For 14 patients who had PR or SD, RT improved the response to CR in six patients $(42.9 \%)$. RT was administered with a median dose of 50.0 Gy (range, 20.0 to 56.0 Gy). Six patients were irradiated using conventional two-dimensional techniques, eight patients received intensity-modulated radiotherapy, and two patients received three-dimensional conformal radiotherapy.

Treatment response of different treatment modalities in NUAT-NKTCL and UAT-NKTCL patients were shown in S3 Table. Among the 90 NUAT-NKTCL patients who received anti-tumor treatment, $C R, P R, S D$, and progressive disease were observed after primary treatment in $39(43.3 \%), 13$ $(14.4 \%), 6(6.7 \%)$, and $28(31.1 \%)$ patients, respectively and unknown in 4 patients (4.4\%). The CR rate between patients treated with ASP-containing and ASP-absent regimens was not statistically different ( $48.3 \%$ vs. $38.5 \%, \mathrm{p}=0.398$ ). The overall response rate (ORR) of the ASP-containing regimens tended to be higher than ASP-absent regimens, although the difference was not statistically different $(66.7 \%$ vs. $46.2 \%$, 
Table 4. Multivariate analysis of prognostic factors in patients with NK/ T-cell lymphoma of non-upper aerodigestive tract

\begin{tabular}{|c|c|c|c|c|}
\hline \multirow{2}{*}{ Variable } & \multicolumn{2}{|l|}{ OS } & \multicolumn{2}{|l|}{ PFS } \\
\hline & $\mathrm{HR}(95 \% \mathrm{CI})$ & p-value & HR $(95 \%$ CI $)$ & p-value \\
\hline Age (> 60 yr vs. $\leq 60$ yr $)$ & - & - & - & - \\
\hline ECOG score ( $\geq 2$ vs. $0-1$ ) & - & - & - & - \\
\hline Stage (III-IV vs. I-II) & - & - & - & - \\
\hline B symptoms (yes vs. no) & $1.984(1.005-3.916)$ & 0.048 & $2.040(1.137-3.661)$ & 0.017 \\
\hline LDH (elevated vs. normal) & $2.147(1.067-4.319)$ & 0.032 & - & - \\
\hline Extranodal sites involvement ( $\geq 2$ vs. $0-1$ ) & - & - & - & - \\
\hline Presence of hemophagocytosis & - & - & - & - \\
\hline
\end{tabular}

The hazard ratio gives the increase in risk for each unit increase for the continuous variables and gives the increased risk relative to the reference category for the categorical variables. NK, natural killer; OS, overall survival; PFS, progression-free survival; HR, hazard ratio; CI, confidence interval; ECOG, Eastern Cooperative Oncology Group; LDH, lactate dehydrogenase.

$\mathrm{p}=0.074$ ). Receiving $\mathrm{RT}$ as part of the primary treatment was associated with a significantly higher ORR $(80.8 \%$ vs. $51.7 \%$, $\mathrm{p}=0.011)$, but not a significantly higher $\mathrm{CR}$ rate $(57.7 \%$ vs. $40.0 \%, \mathrm{p}=0.130$ ).

\section{Survival and prognostic factors}

The median follow-up time was 26.3 months (range, 0.2 to 250.4 months) for all surviving individuals. At the end of last follow-up, 49 NUAT-NKTCL patients (53.3\%) and 213 UATNKTCL patients (32.0\%) had died. The 5-year OS for NUATNKTCL and UAT-NKTCL were $34.7 \%$ and $64.2 \%$, respectively, which was significantly different $(\mathrm{p}<0.001)$ (Fig. 1). And the 5-year PFS rates for NUAT-NKTCL and UAT-NKTCL were $56.1 \%$ and $20.2 \%$, respectively, which was also significantly different $(\mathrm{p}<0.001)$ (Fig. 1$)$. The median OS and PFS duration for NUAT-NKTCL were 30.9 and 12.7 months, respectively, while the median OS and PFS duration for NUAT-NKTCL were not reached and 95.4 months.

Results of univariate analysis in NUAT-NKTCL are summarized in Table 3. We found that patients with B symptoms, elevated serum $L D H$, extranodal sites involvement $\geq 2$, presence of hemophagocytosis, IPI score $\geq 3$ or PINK score $\geq 3$ (Fig. 2A and B) had significantly worse OS, whereas those with B symptoms and presence of hemophagocytosis had significantly worse PFS, compared with their counterparts (Table 3). Different from UAT-NKTCL, Ann Arbor stage system failed to predict OS or PFS in NUAT-NKTCL (Fig. 3). Patients who received RT had a significantly longer PFS (hazard ratio [HR], $0.478 ; 95 \%$ confidence interval [CI], 0.265 to $0.861 ; \mathrm{p}=0.014)$ but not a significantly better OS (HR, 0.634 ; $95 \% \mathrm{CI}, 0.328$ to $1.224 ; \mathrm{p}=0.174)$. Patients treated with chemotherapeutic regimens containing ASP tended to have a better OS (HR, 0.601; 95\% CI, 0.332 to 1.089; $\mathrm{p}=0.093$ ) and PFS (HR,
0.673; 95\% CI, 0.397 to 1.142; $\mathrm{p}=0.142$ ), although the differences were not significantly different. Patients who achieved $\mathrm{CR}$ after primary treatment had significantly better OS and PFS than those did not, both with $\mathrm{p}$-value of $<0.001$.

In Table 4, multivariate analysis showed that $\mathrm{B}$ symptoms (HR, 1.984; 95\% CI, 1.005 to 3.916; $\mathrm{p}=0.048$ ) and elevated LDH levels (HR, 2.147; 95\% CI, 1.067 to 4.319; $\mathrm{p}=0.032$ ) were both independently associated with worse OS. Presence of $B$ symptoms independently correlated with worse PFS (HR, $2.040 ; 95 \%$ CI, 1.137 to $3.661 ; \mathrm{p}=0.017$ ).

\section{Discussion}

NUAT-NKTCL was considered as a unique subtype of NKTCL since heterogeneity was found between UAT-NKTCL and NUAT-NKTCL in several previous studies [2-10], in aspects of clinical characteristics, treatment response, prognostic factors, and longterm survival. In this retrospective single-center study, we comprehensively analyzed clinical data of patients diagnosed with NUAT-NKTCL from 2001 to 2017, aiming to provide a better understanding of this entity.

The most common site of NUAT-NKTCL was the skin and soft tissue $(\mathrm{n}=42,45.7 \%)$, which was consistent with results of published literature [3,5,7-10,16-19]. NUAT-NKTCL patients had a male to female ratio of 1.7:1 and the median age of 35 years at diagnosis with $13.0 \%$ were older than 60 , which were similar to UAT-NKTCL patients. Similarly, several studies had found no obvious difference between UAT-NKTCL and NUAT-NKTCL in terms of sex and age $[2,4,7,8,10,20]$. Overall, the NUAT-NKTCL cohort in this study presented with a high-risk clinical profile. Consistent with results of 
previous studies, we found that more NUAT-NKTCL patients had ECOG scores $\geq 2(42.4 \%)[2-5,10]$, involvement of regional lymph nodes $(51.7 \%)[3,5,7]$, involvement of distant lymph nodes $(28.1 \%), \geq 2$ extranodal sites $(44.0 \%)$ [3,5,7,9], involvement of bone marrow (11.0\%), elevated serum LDH $(55.4 \%)[2-4,7,9,10]$, IPI score $\geq 3(50.0 \%)[4-7,9,10]$, PINK score $\geq 3(36.3 \%)$ [21], and Ann Arbor stage III/IV (64.8\%) [2-10,22]. The median of Ki-67 index was 70\% for NUATNKTCL patients, which was significantly higher than UATNKTCL $(60 \%)$ and indicated highly malignant features $[8,9]$. In conclusion, patients with NUAT-NKTCL had worse performance status, more unfavorable prognostic factors, and more advanced stage.

Ann Arbor stage was proved to be an independent prognostic factor of long term survival for NKTCL patients in most studies. However, we found that Ann Arbor stage could not predict OS or PFS for NUAT-NKTCL patients. An explanation for this phenomenon is that nearly half of cases originated at the skin and soft tissues, which were often staged as stage III or IV in the Ann Arbor stage system although some had relatively favorable clinical outcomes. Published researches had confirmed the prognostic values of IPI score and PINK score in NKTCL [21,23]. Although both of them could not significantly discriminate every risk subgroup, high-intermediate risk group (IPI score=3) and high risk group (IPI score $=4,5$ ) of IPI, and 3 or 4 risk factors of PINK predicted worse OS.

Approximately 70\% of NUAT-NKTCL patients were treated with $\mathrm{CT}$ alone as primary treatment and only about $30 \%$ received RT, which was different from UAT-NKTCL (only $29.0 \%$ accepted CT alone, $\mathrm{p}<0.001$ ) and in accord with results of previous studies $[2,3,7,22]$. The proportion of RT administration between NUAT-NKTCL patients with limited and advanced-stage disease was also significantly different $(46.9 \%$ vs. $18.6 \%, \mathrm{p}<0.001)$. More advanced stage patients of NUAT-NKTCL somehow explained the much lower proportion of RT administration, compared with UATNKTCL. On the other hand, a curative dose of RT (usually $50 \mathrm{~Gy}$ ) may not be suitable for some specific sites of primary lesion in NUAT-NKTCL, such as the lungs, the GI tract, etc. According to present evidences, UAT-NKTCL is sensitive to RT and has a CR rate of 70\% to $90 \%$ for limited-stage disease [24-28]. We also found that a CR rate of 90.5\% (362 of 400) in limited-stage UAT-NKTCL patients whose treatment response could be evaluated. However, the CR rate in limitedstage NUAT-NKTCL patients was only 66.7\% (10 of 15), which was significantly lower ( $\mathrm{p}=0.003$ ). Besides, receiving RT as part of the primary treatment did not significantly influence the CR rate or OS, although it did improve the ORR and PFS. Considering the above results, the sensitivity of NUAT-NKTCL to irradiation may need further exploring. The CR rate and the ORR were $40.0 \%$ and $51.7 \%$ respectively in patients received $\mathrm{CT}$ alone as primary treatment. Patients treated with ASP-containing regimens tended to have higher ORR and CR rate. CT should still be recommended in most NUAT- NKTCL patients considering the high percentage of advanced-stage disease.

Univariate analysis found that patients with B symptoms, elevated serum LDH levels, extranodal sites involvement $\geq 2$, and the presence of hemophagocytosis had significantly worse OS, whereas those with B symptoms or presence of hemophagocytosis had significantly worse PFS. High IPI scores $(\geq 3)$ and high PINK scores $(\geq 3)$ both correlate with worse OS. Administration of RT in primary therapy significantly improved PFS but not OS. ASP-containing regimens tended to be associated with better OS and PFS. Patients achieved CR after primary treatment had significantly longer OS and PFS. According to previous studies, these prognostic factors are closely relevant to each other, which might result in misleading interpretations of their prognostic values. Thus we subsequently conducted a multivariate analysis to find independent prognostic factors for NUAT-NKTCL. We found that B symptoms and elevated serum LDH levels were significantly correlated with worse OS, and presence of B symptoms also predicted shorter PFS.

The 5-year OS for NUAT-NKTCL was $34.7 \%$ and the median OS duration was 30.9 months, which were better than results of previous studies $[2-10,16,29,30]$. This might be attributed to the development of therapeutic methods and the improvement of supportive care during recent years. However, long-term survival of NUAT-NKTCL was still much worse than UAT-NKTCL.

There are limitations due to the retrospective nature of our study. Patients with lesions located in both the UAT and NUAT sites at initial presentation were classified as UATNKTCL in our analysis. Some patients were treated with diverse chemotherapeutic regimens in primary treatment. Moreover, some important data were not available for every patient, such as serum LDH, plasma EBV DNA copy number, and so on. Further efforts should be made to focus on the improvement of treatment and survival for NUAT-NKTCL in large scale multicenter studies.

In conclusion, NUAT-NKTCL is a distinctive subtype of NKTCL in many aspects. Patients with NUAT-NKTCL have relatively poorer performance status, more unfavorable prognostic factors, more advanced stage, and poorer prognosis.

\section{Electronic Supplementary Material}

Supplementary materials are available at Cancer Research and Treatment website (https: // www.e-crt.org). 


\section{Conflicts of Interest}

Conflict of interest relevant to this article was not reported.

\section{Acknowledgments}

This work was supported by grants from National Natural Science Foundation of China (No. 81502573, No.81600153, and No. 81700196), Fundamental Research Funds for the Central Universities (No. 17ykpy77), and Medical Scientific Research Foundation of Guangdong Province of China (No. A2016013).

\section{References}

1. Swerdlow SH, Campo E, Pileri SA, Harris NL, Stein H, Siebert $\mathrm{R}$, et al. The 2016 revision of the World Health Organization classification of lymphoid neoplasms. Blood. 2016;127:237590.

2. Au WY, Weisenburger DD, Intragumtornchai T, Nakamura S, Kim WS, Sng I, et al. Clinical differences between nasal and extranasal natural killer/T-cell lymphoma: a study of 136 cases from the International Peripheral T-Cell Lymphoma Project. Blood. 2009;113:3931-7.

3. Kim TM, Lee SY, Jeon YK, Ryoo BY, Cho GJ, Hong YS, et al. Clinical heterogeneity of extranodal NK/T-cell lymphoma, nasal type: a national survey of the Korean Cancer Study Group. Ann Oncol. 2008;19:1477-84.

4. Lim ST, Hee SW, Quek R, Lim LC, Yap SP, Loong EL, et al. Comparative analysis of extra-nodal NK/T-cell lymphoma and peripheral T-cell lymphoma: significant differences in clinical characteristics and prognosis. Eur J Haematol. 2008;80: 55-60.

5. Suzuki R, Suzumiya J, Yamaguchi M, Nakamura S, Kameoka J, Kojima H, et al. Prognostic factors for mature natural killer (NK) cell neoplasms: aggressive NK cell leukemia and extranodal NK cell lymphoma, nasal type. Ann Oncol. 2010;21: 1032-40.

6. Pongpruttipan T, Sukpanichnant S, Assanasen T, Wannakrairot $\mathrm{P}$, Boonsakan $\mathrm{P}$, Kanoksil W, et al. Extranodal NK/ T-cell lymphoma, nasal type, includes cases of natural killer cell and alphabeta, gammadelta, and alphabeta/gammadelta T-cell origin: a comprehensive clinicopathologic and phenotypic study. Am J Surg Pathol. 2012;36:481-99.

7. Jo JC, Yoon DH, Kim S, Lee BJ, Jang YJ, Park CS, et al. Clinical features and prognostic model for extranasal NK/T-cell lymphoma. Eur J Haematol. 2012;89:103-10.

8. Li S, Feng X, Li T, Zhang S, Zuo Z, Lin P, et al. Extranodal NK/T-cell lymphoma, nasal type: a report of 73 cases at MD Anderson Cancer Center. Am J Surg Pathol. 2013;37:14-23.

9. Bai B, Huang HQ, Cai QC, Fan W, Wang XX, Zhang X, et al. Predictive value of pretreatment positron emission tomography / computed tomography in patients with newly diagnosed extranodal natural killer/T-cell lymphoma. Med Oncol. 2013; 30:339.

10. Lee J, Park YH, Kim WS, Lee SS, Ryoo BY, Yang SH, et al. Extranodal nasal type NK/T-cell lymphoma: elucidating clin- ical prognostic factors for risk-based stratification of therapy. Eur J Cancer. 2005;41:1402-8.

11. Kim GE, Lee SW, Chang SK, Park HC, Pyo HR, Kim JH, et al. Combined chemotherapy and radiation versus radiation alone in the management of localized angiocentric lymphoma of the head and neck. Radiother Oncol. 2001;61:261-9.

12. Kim BS, Kim TY, Kim CW, Kim JY, Heo DS, Bang YJ, et al. Therapeutic outcome of extranodal NK/T-cell lymphoma initially treated with chemotherapy: result of chemotherapy in NK/T-cell lymphoma. Acta Oncol. 2003;42:779-83.

13. Kim WS, Song SY, Ahn YC, Ko YH, Baek CH, Kim DY, et al. $\mathrm{CHOP}$ followed by involved field radiation: is it optimal for localized nasal natural killer/T-cell lymphoma? Ann Oncol. 2001;12:349-52.

14. Cheson BD, Fisher RI, Barrington SF, Cavalli F, Schwartz LH, Zucca E, et al. Recommendations for initial evaluation, staging, and response assessment of Hodgkin and non-Hodgkin lymphoma: the Lugano classification. J Clin Oncol. 2014;32: 3059-68.

15. Tse E, Kwong YL. How I treat NK/T-cell lymphomas. Blood. 2013;121:4997-5005.

16. Lee J, Suh C, Park YH, Ko YH, Bang SM, Lee JH, et al. Extranodal natural killer T-cell lymphoma, nasal-type: a prognostic model from a retrospective multicenter study. J Clin Oncol. 2006;24:612-8

17. Ren YL, Nong L, Zhang S, Zhao J, Zhang XM, Li T. Analysis of 142 Northern Chinese patients with peripheral T/NK-Cell lymphomas: subtype distribution, clinicopathologic features, and prognosis. Am J Clin Pathol. 2012;138:435-47.

18. Huang JJ, Zhu YJ, Xia Y, Zhao W, Lin TY, Jiang WQ, et al. A novel prognostic model for extranodal natural killer/T-cell lymphoma. Med Oncol. 2012;29:2183-90.

19. Cai Q, Luo X, Liang Y, Rao H, Fang X, Jiang W, et al. Fasting blood glucose is a novel prognostic indicator for extranodal natural killer/T-cell lymphoma, nasal type. Br J Cancer. 2013; 108:380-6.

20. Vose J, Armitage J, Weisenburger D; International T-Cell Lymphoma Project. International peripheral T-cell and natural killer/T-cell lymphoma study: pathology findings and clinical outcomes. J Clin Oncol. 2008;26:4124-30.

21. Kim SJ, Yoon DH, Jaccard A, Chng WJ, Lim ST, Hong H, et al. A prognostic index for natural killer cell lymphoma after non- 
anthracycline-based treatment: a multicentre, retrospective analysis. Lancet Oncol. 2016;17:389-400.

22. Chauchet A, Michallet AS, Berger F, Bedgedjian I, Deconinck $\mathrm{E}$, Sebban $\mathrm{C}$, et al. Complete remission after first-line radiochemotherapy as predictor of survival in extranodal NK/T cell lymphoma. J Hematol Oncol. 2012;5:27.

23. Chim CS, Ma SY, Au WY, Choy C, Lie AK, Liang R, et al. Primary nasal natural killer cell lymphoma: long-term treatment outcome and relationship with the International Prognostic Index. Blood. 2004;103:216-21.

24. Li YX, Yao B, Jin J, Wang WH, Liu YP, Song YW, et al. Radiotherapy as primary treatment for stage IE and IIE nasal natural killer/T-cell lymphoma. J Clin Oncol. 2006;24:181-9.

25. Wang H, Li YX, Wang WH, Jin J, Dai JR, Wang SL, et al. Mild toxicity and favorable prognosis of high-dose and extended involved-field intensity-modulated radiotherapy for patients with early-stage nasal NK/T-cell lymphoma. Int J Radiat Oncol Biol Phys. 2012;82:1115-21.

26. Wang ZY, Li YX, Wang WH, Jin J, Wang H, Song YW, et al. Primary radiotherapy showed favorable outcome in treating extranodal nasal-type NK/T-cell lymphoma in children and adolescents. Blood. 2009;114:4771-6.

27. Kim GE, Cho JH, Yang WI, Chung EJ, Suh CO, Park KR, et al. Angiocentric lymphoma of the head and neck: patterns of systemic failure after radiation treatment. J Clin Oncol. 2000;18: 54-63.

28. Kim K, Chie EK, Kim CW, Kim IH, Park CI. Treatment outcome of angiocentric T-cell and NK/T-cell lymphoma, nasal type: radiotherapy versus chemoradiotherapy. Jpn J Clin Oncol. 2005;35:1-5.

29. Lee J, Au WY, Park MJ, Suzumiya J, Nakamura S, Kameoka J, et al. Autologous hematopoietic stem cell transplantation in extranodal natural killer/T cell lymphoma: a multinational, multicenter, matched controlled study. Biol Blood Marrow Transplant. 2008;14:1356-64.

30. Ai WZ, Chang ET, Fish K, Fu K, Weisenburger DD, Keegan TH. Racial patterns of extranodal natural killer/T-cell lymphoma, nasal type, in California: a population-based study. Br J Haematol. 2012;156:626-32. 